\title{
Gut Microbiota-Brain Axis Modulation by a Healthier Microbiological Microenvironment : Facts and Fictions
}

\author{
Uday C Ghoshal \\ Department of Gastroenterology, Sanjay Gandhi Post Graduate Institute of Medical Sciences, Lucknow, India
}
Article: Bifidobacterium longum and Lactobacillus helveticus synergistically suppress stress-related visceral hypersensitivity through hypothalamic-pituitary-adrenal axis modulation
Ait-Belgnaoui A, Payard I, Rolland C, et al
(J Neurogastroenterol Motil 2018;24:138-146)

Functions of every organ and system of the body need to be perfectly regulated for the maintenance of the milieu interior; this is performed by a wireless (endocrine) system and a wired (nervous) system in the body. Interestingly, the communication between the brain and the gut is bi-directional; though, the brain controls the gut to a large extent, the gut also influences the brain functions. In recent years, it has been realized that the gut microbiota, which is the largest organ of the human body, influences the brain functions. ${ }^{1,2}$ However, this is not entirely a new concept. As early as pre-2nd century CE, in the Charaka Samhita, an Indian book on medicine, it was mentioned that the human being is a microcosmic replica of the universe, ${ }^{3}$ and the 3 humors (Vayu or gas, Pitta or bile, and Kapha or mucus) are responsible for most ailments. ${ }^{4}$ It is interesting to know how the gastrointestinal tract in general and its microbiota, in particular, is being unraveled by the scientists in recent times.

Gut microbial modulation of the brain function is best demonstrated by the well-known effects of gut-microbiota directed antibiotic treatment in patients with hepatic encephalopathy. Moreover, in recent times, several animal and human experiments also demonstrated the role of gut microbiota in influencing brain function. In a study by De Palma et al, ${ }^{5}$ germ-free mice were shown to have dysregulated hypothalamic pituitary stress response, altered brainderived neurotrophic factors (BDNF) in the hippocampus, altered neurotransmission, altered tryptophan availability and metabolism, and reduced anxiety. In another experimental study, antibiotic treatment of $\mathrm{BALB} / \mathrm{c}$ mice resulted in increase in Firmicutes phylum dominated by Lactobacillus, sulfate-reducing bacterial species, and decrease in $\gamma$-Proteobacteria and Bacteroidetes, which was associated with increase in hippocampal BDNF and autonomic-independent anxiolytic behavior. ${ }^{6}$

How does gut microbiota alter brain function? In a study on wild mice separated from their mothers, which is well known to induce anxiety, it was found to be associated with altered gut-microbial colonization, long-lasting hyperactivity of the hypothalamic pituitary-axis, visceral hypersensitivity, altered cholinergic activity in the gut, and increased intestinal permeability. ${ }^{5,7}$ Interestingly, treatment

Received: December 26, 2017 Revised: None Accepted: December 26, 2017

(c) This is an Open Access article distributed under the terms of the Creative Commons Attribution Non-Commercial License (http://creativecommons. org/licenses/by-nc/4.0) which permits unrestricted non-commercial use, distribution, and reproduction in any medium, provided the original work is properly cited.

*Correspondence: Uday C Ghoshal, MD, DNB, DM, FACG, RFF, FNAMS

Department of Gastroenterology, Sanjay Gandhi Postgraduate Institute of Medical Science, Lucknow 226014, India

Tel: +91-522-249-4406, Fax: +91-522-2668017, E-mail: udayghoshal@gmail.com 
with probiotics reduced the development of these effects. ${ }^{5,7}$ Moreover, the role of gut microbiota in causing these pathophysiological effects was also evidenced by the fact that separation of germ-free mice from their mothers showed no such effect. ${ }^{5,7}$ Corticotrophinreleasing factor from the hypothalamus stimulates the release of adrenocorticotrophic hormone from the anterior pituitary that stimulates secretion of glucocorticoids (a stress hormone like adrenaline) from the adrenal glands. ${ }^{8}$ Hypothalamic-pituitary adrenal (HPA) axis determines stress response in animals and humans. ${ }^{8}$ Recently, evidence is emerging showing that gut microbiota may influence the HPA axis. Studies showed that in the absence of enteric microbiota, gut toll-like receptors (TLR), which recognize the microbiota-associated molecular-pattern to mount host response, was low and intact TLR4 signaling was required for plasma corticosterone release. ${ }^{9}$ It has also been shown that TLR4 knockout mouse did not respond to lipopolysaccharide of Gram-negative bacteria with activation of the HPA axis. ${ }^{9}$ Inducing stress in germ-free mice by restraining them resulted in higher corticosterone and adrenocorticotrophic hormone release as compared to control mice, who had microbes in their gut that was specific pathogens free (SPF); ${ }^{9}$ such aberrant response was partially reversed by colonization with fecal matter from SPF animals and fully by association with Bifidobacterium infantis, which is a probiotic bacteria. ${ }^{9}$

Another mechanism by which gut microbiota may influence stress response in the brain by altering CNS biochemistry may be related to low-grade inflammation of the gut due to microbial dysbiosis. ${ }^{10}$ Animals such as mice have a natural tendency to hide in darkness for security, but also need to explore for foods in the illuminated areas. In light and dark box experiments, the proportion of time mice spent in the dark box as compared to light box (both are inter-connected) gives an estimate of anxiety; ${ }^{11}$ anxious animals spent relatively more time in darker areas. Similarly, mice are placed on a table and the latent period it takes to jump down from it gives an idea about its anxiety level; anxious animals take a longer time to jump down as compared to less anxious animals. ${ }^{12}$ Bercik et $\mathrm{al}^{10}$ reported an experimental study on mouse models on various interventions (administration of budesonide, etanercept, probiotics, and surgical vagotomy) that potentially blocked inflammation or caused inflammation following gut infection with Trichuris muris. They found that latency to step-down from the table was longest (denotes higher level of anxiety) with placebo with $T$. muris infection as compared to most other interventions mentioned above that block gut inflammation or altered immune response; similarly, time in the light box was shortest (denotes higher level of anxiety) with placebo with $T$. muris infection as compared to most other interventions. ${ }^{10}$
Anxiety was associated with lower BDNF messenger ribonucleic acid in the hippocampus and greater mononuclear infiltrate scores. ${ }^{10}$

Though quite a few animal studies demonstrated the role of gut microbiota in brain function, data from human studies are somewhat scanty. In a recent study, germ-free mice were colonized by fecal microbiota from 8 patients with diarrhea-predominant irritable bowel syndrome (IBS) and 5 healthy controls. ${ }^{13}$ Fecal microbiota analysis of mice showed that it resembled the microbiota of the donor. Authors found that mice receiving microbiota from patients with IBS were more anxious (demonstrated by latency to step-down from the table and time spent in the light box) than those receiving microbiota from healthy subjects; ${ }^{13}$ Intestinal transit, paracellular gut permeability, and the molecular markers of gut inflammation were also greater among the former group of mice. ${ }^{13}$

Though the above-mentioned mechanistic studies are important in a scientific perspective, clinicians and patients are interested in knowing whether therapeutic interventions targeting the gut microbiota would have any clinically meaningful alteration in brain functions that may benefit the patients. However, we need to wait for a long time before this becomes reality in clinical practice except for gut microbiota alteration benefiting patients with hepatic encephalopathy. However, a few recent pilot studies provide some rays of hope. A small study on 10 healthy subjects receiving probiotic bacteria Lactobacillus helveticus R0052 and Bifidobacterium longum $\mathrm{R} 0175$ showed that probiotic bacteria improved perceived stress scale and hospital anxiety and depression scale more than placebo. ${ }^{14}$ In another elegant study, 36 healthy females underwent restingstate functional magnetic resonance imaging (fMRI) and response to stress by showing emotional faces was studied; subsequently, they were subjected to ingestion of fermented milk product with probiotic $(\mathrm{n}=11)$, non-fermented milk $(\mathrm{n}=12)$ for 4 weeks and no intervention $(\mathrm{n}=13) .{ }^{15}$ Resting state $\mathrm{fMRI}$ and response to stress (by showing emotional faces) studies were repeated. Authors found that one month intake of fermented milk product with probiotic in healthy women was associated with alteration in activity of brain regions that control central processing of emotion and sensation. In this issue of the Journal of Neurogastroenterology and Motility, ${ }^{16}$ authors from France, Canada, Sweden studied the comparative effects of treatment of mice with different probiotics (B. longum and $L$. helveticus) for 2 weeks on the perception of visceral pain induced by colorectal distension after chronic stress and on the activity of the HPA axis. The authors concluded that the combination of the 2 probiotics showed greater efficacy than either alone in regulating glucocorticoid induced negative feedback on the HPA axis; hence, they suggested that this observation may have implication in the 
treatment of stress-induced visceral pain. It was also observed that glucocorticoid receptor gene expression in the hypothalamus and hippocampus, which is under-expressed during chronic stress, was restored by pre-treatment with probiotics. This study is an important contribution to the understanding of the effect of therapeutic modulation of gut microbiota using specific strains of probiotics on brain function. However, in spite of this study, the journey paving way to clinical application of such research is long as there is lack of high quality randomized double-blind placebo-controlled trials in human subjects, including those with functional gastrointestinal disorders such as IBS, comparison of multiple strains and dose of the probiotics to understand which strains work at what dose, relationship between response and type of gut microbiota, predictors of response, comparison between various duration of treatment, and duration of persistence of response following treatment discontinuation. In conclusion, though several animal studies including the current one showed the efficacy of some probiotic formulations to influence CNS functions, these need to pave the way towards more and more research with clinical studies as extrapolating animal studies to clinical application suffers from limitations though it may offer possibilities. This reminds us of Albert Einstein's saying “To raise new questions, new possibilities, to regard old problems from a new angle, requires creative imagination and marks a real advance in science."

\section{References}

1. Quigley EMM. Microbiota-brain-gut axis and neurodegenerative diseases. Curr Neurol Neurosci Rep 2017;17:94.

2. Ghoshal UC, Ghoshal U. Small intestinal bacterial overgrowth and other intestinal disorders. Gastroenterol Clin North Am 2017;46:103-120.

3. Glucklich A. The Strides of Vishnu: Hindu Culture in Historical Perspective. New York: Oxford University Press 2008:141-142.

4. Dasgupta S. A History of Indian philosophy. Cambridge: Cambridge University Press 1922;1:325-339.

5. De Palma G, Collins SM, Bercik P. The microbiota-gut-brain axis in functional gastrointestinal disorders. Gut Microbes 2014;5:419-429.
6. Bercik P, Denou E, Collins J, et al. The intestinal microbiota affect central levels of brain-derived neurotropic factor and behavior in mice. Gastroenterology 2011;141:599-609, e1-e3.

7. De Palma G, Blennerhassett P, Lu J, et al. Microbiota and host determinants of behavioural phenotype in maternally separated mice. Nat Commun 2015;6:7735.

8. Vachon-Presseau E. Effects of stress on the corticolimbic system: implications for chronic pain. Prog Neuropsychopharmacol Biol Psychiatry Published Online First: 25 Oct 2017. doi: 10.1016/j.pnpbp.2017.10.014.

9. Clarke G, Stilling RM, Kennedy PJ, Stanton C, Cryan JF, Dinan TG. Minireview: gut microbiota: the neglected endocrine organ. Mol Endocrinol 2014;28:1221-1238.

10. Bercik P, Verdu EF, Foster JA, et al. Chronic gastrointestinal inflammation induces anxiety-like behavior and alters central nervous system biochemistry in mice. Gastroenterology 2010;139:2102-2112, e1 .

11. Crawley J, Goodwin FK. Preliminary report of a simple animal behavior model for the anxiolytic effects of benzodiazepines. Pharmacol Biochem Behav 1980;13:167-170.

12. York JM, Blevins NA, Baynard T, Freund GG. Mouse testing methods in psychoneuroimmunology: an overview of how to measure sickness, depressive/anxietal, cognitive, and physical activity behaviors. Methods Mol Biol 2012;934:243-276.

13. De Palma G, Lynch MD, Lu J, et al. Transplantation of fecal microbiota from patients with irritable bowel syndrome alters gut function and behavior in recipient mice. Sci Transl Med 2017;9:eaaf6397.

14. Messaoudi M, Violle N, Bisson JF, Desor D, Javelot H, Rougeot C. Beneficial psychological effects of a probiotic formulation (Lactobacillus helveticus R0052 and Bifidobacterium longum R0175) in healthy human volunteers. Gut Microbes 2011;2:256-261.

15. Tillisch K, Labus J, Kilpatrick L, et al. Consumption of fermented milk product with probiotic modulates brain activity. Gastroenterology 2013;144:1394-1401, e1-e4.

16. Ait-Belgnaoui A, Payard I, Rolland C, et al. Bifidobacterium longum and Lactobacillus helveticus synergistically suppress stress-related visceral hypersensitivity through hypothalamic-pituitary-adrenal axis modulation. J Neurogastroenterol Motil 2018;24:138-146.

\section{Financial support: None.}

\section{Conflicts of interest: None.}

